dienstliche Erklärung handeln, die den Schutz der Wissenschaftsfreiheit wohl auf keinen Fall genösse. Ich würde überdies faktisch einräumen, daß Juristen, noch dazu offensichtlich geschichtslose Köpfe, darüber zu entscheiden haben, was Wissenschaft ist und was nicht. Das ist einem Hochschullehrer nicht zumutbar. Abschließend: Es war 1972 möglich, meine Dienstenthebung auf Grund des selbstverständlich unbegründeten - Vorwurfs nach $\$ I 29 StGB als individuelles Pech, oder meinetwegen: als persönlichen Makel anzusehen; nicht anders als uneheliche Schwangerschaften in der bürgerlichen Gesellschaft: sie kommen vor, aber Gottseidank sehr selten, die Familien sind nicht betroffen. Der jüngste Versuch, einen Hochschullehrer über die juristische Uberprüfung seiner Publikationen dem >Radikalen`-Erlaß der Länder-Ministerpräsidenten zu subsumieren, bei äußerst restriktiver Auslegung des Art. s GG Abs. 3, ist doch von allgemeiner Bedeutung. Daher diese kurze Mitteilung, der eine gründliche politische, verfassungs- und beamtenrechtliche Prüfung bald nachfolgen sollte. Die Autoren der "Staatsfeinde ${ }^{6} \mathbf{6}$ - Alfred Krovoza und ich - haben sich nicht geirrt: auch Probleme der wissenschaftstheoretischen Kontroverse werden an Instrumente der Herrschaftssicherung delegiert.

Peter Brückner

\title{
Berufsverbot durch Verfassungsschutz
}

Im Zusammenhang mit den Berufsverboten, die sich seit dem Ministerpräsidentenbeschluß vom Januar 1972 häufen, werden in Presse und Rundfunk immer wieder die Ämter für Verfassungsschutz (ÄfV) und die Politische Polizei als Lieferanten von Denunziationen zur Begründung der Berufsverbote genannt. Anhand einiger exemplarischer Beispiele soll im folgenden versucht werden, die Prakriken dieser der Kontrolle der Ơffentlichkeit weitgehend entzogenen Staatsschutzorgane zu erhellen. ${ }^{1}$

Zunächst einige Anmerkungen zu Struktur und Aufgabe der AfV. In der BRD existiert ein Bundesamt für Verfassungsschutz (BfV), das der Aufsicht des Bundesinnenministeriums untersteht. Zusätzlich besteht in jedem Bundesland ein Landesamt für Verfassungsschutz (LfV), das meist dem Innenministerium eingefügt und das gegenüber dem BfV relativ selbständig ist. Nach dem Bundesverfassungsschutzgesetz von 1950 sollten die AfV Nachrichten über Bestrebungen sammeln und auswerten, die die verfassungsmäßige Ordnung der BRD beeinträchtigen könnten. Durch intensive "Reformpolitik « sind die Befugnisse der AfV im Rahmen der Notstandsverfassung 1968 auf das Abhören von Telefongesprächen und das Ablichten von Briefen und 1972 auf die Uberwachung von Spionen und Ausländern erweitert worden: "Aufgabe des BfV und anderer dazu bestimmter Behörden ist die Sammlung und Auswertung von Auskünften, Nachrichten und sonstigen Unterlagen über I.) Bestrebungen, die gegen die freiheitlich demokratische Grundordnung, den Bestand und die Sicherheit des Bundes oder eines Landes gerichtet sind oder eine ungesetzliche Beeinträchtigung der

\footnotetext{
6 Politik* Bd. 40, Wagenbach 1972.

1 Umfassender sind die Praktiken des VS dargestellt in: D. Damm, So arbeitet der VS, Berlin 1970. Hier finden sich auch die Quellennachweise für alle Fälle, deren Publikationsort im folgenden nicht extra angegeben wird.
} 
Amtsführung von Mitgliedern verfassungsmäßiger Organe des Bundes oder eines Landes zum Ziel haben, 2.) sicherheitsgefährdende oder geheimdienstliche Tätigkeiten im Geltungsbereich dieses Gesetzes für eine fremde Macht, 3.) die Bestrebungen im Bundesgebiet, die durch Anwendung von Gewalt oder darauf gerichtete Vorbereitungsverhandlungen auswärtige Belange der Bundesrepublik Deutschland gefährden. «²

Polizeiliche oder Kontrollbefugnisse stehen den Aff nicht zu. Sie können, jedenfalls theoretisch, nur über die Staatsanwaltschaft bz.w. die PolitischePolizei exekutiv tätig werden. Was die finanziellen Mittel anbetrifft, so konnte auch hier durch Sozialdemokratische »Reformpolitik « der Etat, der 1969 29,9 Mill. DM nur für das BfV umfaßte, um über hundert Prozent auf 62, r Mill. DM 1973 gesteigert werden. ${ }^{3}$ Dazu kommen die Etats der L.fV und die nicht offen ausgewiesenen Mittel.

Der VS ist zur gegenseitigen Information und Zusammenarbeit mit der Politischen Polizei verpflichtet, die "für alle Straftaten, die gegen den Bestand der Bundesrepublik oder eines ihrer Länder oder gegen die verfassungsnüßige Ordnung gerichtet sind «, zuständig ist. Neben den $\mathrm{AfV}$, der Politischen Polizei und der 300-köpfigen Sicherungsgruppe Bonn operieren noch der Bundesnachrichtendienst (BND) und der Militärische Abwehrdienst (MAD) für die Aufrechterhaltung der "verfassungsmäßigen Ordnung « - einmal abgesehen von den zahlreichen westlichen Geheimdiensten, insonderheit dem CIA.

Während der MAD eigentlich nur für militärische Sicherheitsfragen und der BND nur für Spionage im Ausland, vornehm »Auslandsaufklärung « genannt, zuständig sein sollen, sieht, wie bei den $\mathrm{AfV}$, auch hier die Praxis etwas anders aus. So erklärte 1972 der damalige Kanzleramtsminister Ehmke, daß der BND bisher gesetzeswidrig nachrichtendienstliche Tätigkeit auch im Inland betrieben habe. Ehmke erboste, daß zahlreiche Dossiers von SPD-Politikern angefertigt worden waren sowie die generelle Richtung der BND-Forschungen: so sei noch im Jahr 197 I festgestellt worden, daß eine Außenstelle des Geheimdienstes Material über die SPD sammelte. Dieses Material sei allerdings nicht der BND-Zentrale in München-Pullach, sondern einer wanderen Stelle " zugeleitet worden. ${ }^{4}$ VS, BND und MAD haben weiterhin versucht, Spitzel in linksgerichtete Studentenorganisationen einzuschleusen. Dabei gibt es interessante Kooperationsformen $\mathrm{zw}$ wischen den verschiedensten Bundes- und Landesbehörden, wie etwa folgender Fall beweist, der in einer Sendung des NDR berichtet wurde: "Der Schüler O. hat im Frühjahr 70 sein Abitur eben hinter sich. Zum I. Juli sollte er einrücken. Kurz vorher hat ein V-Mann des Verfassungsschutzes oder MAD ... ihm angeboten, einmal Befreiung vom Wehrdienst und ein monatliches Honorar. Dieser V-Mann hat sich als Mitarbeiter eines Instituts für Gesellschaftsforschung oder so ähnlich in Mainz ausgegeben, und der Zeuge sollte Material für angebliche statistische Erhebungen beschaffen. $O$. hat sich dumm gestellt und ist zum Schein auf das Angebot eingegangen. ${ }^{2-3}$ Tage später kam prompt die Beurlaubung vom Wehrdienst; das Zusammenspiel der Behörden hat also gut funktioniert. $O$. hat dann aber seine Zusage zurückgenommen. Schriftliche Abmachungen gab es nicht; da war man auf Geheimdienstseite gewieft genug, so daß man nichts nachweisen kann ... Die ganze Geschichte spielte sich im Zeitraum von gut einer Woche ab. Weswegen man gerade auf $\mathrm{O}$. gekommen ist, das kann er sich nur so

\footnotetext{
2 Frankfurter Rundschau (FR) vom 19. 6. 1972.

3 FR 24. 3. 1972.

4 FR 22. 9. 1972, 6. 10. I97 I; vgl. dazu auch: Stern 44/1973, S. 200.
} 
erklären, daß die Geheimdienste sich Bundeswehrunwillige herausfischen und die dann mit solchen Angeboten erpressen oder zumindest es versuchen ... Die studentischen Rechercheure ermittelten weiterhin, daß dem Vater des Zeugen, der sich beim Kreiswehrersatzamt Koblenz von der Richtigkeit der Angaben seines Sohnes überzeugen wollte, geantwortet wurde, O. sei auf Anweisung des MAD zurückgestellt worden. Beim Kreiswehrersatzant weiß man natürlich auf Anfrage weder etwas von Weisungen des MAD noch von den besagten rechtswidrigen Zurückstellungen. Auch die Wehrbereichsverwaltung in Wiesbaden ist angeblich ahnungslos. Einem Rechercheur der Mainzer Studentenschaft freilich, der im Beisein von Zeugen unter dem Namen des Spitzels in Koblenz angerufen hatte, wurde von dort mitgeteilt, seine - also des Spitzels - Akte liege wegen einer MAD-Angelegenheit gerade beim Behördenleiter. Nach allem sieht es so aus, als hätten Abschirmdienst und VS, für den die Studenten spitzeln sollten, gemeinsam gearbeitet ... Von einer anderen Bestätigung der Spitzeltätigkeit in der Studentenschaft weiß Michael Bischoff, Asta-Vorsitzender in Mainz, zu berichten. Einem Vorfall, der einem Doktoranden zugestoßen ist, der für die Bewilligung eines Stipendiums einen Gutachter benötigte und auf dessen Fragen, aus Angst vor Ablehung, seine politische Betätigung abstritt. Bischoff: Daraufhin zog der Gutachter ein kleines Dossier, und zwar eine Personalakte, aus seiner Schublade und rechnete diesem unserem Mitglied haarklein vor, daß es Mitglied der linken Fraktion des Mainzer Studentenparlaments war, daß es in der und der Gruppe war, daß es in der und der Gruppe verschiedene Außerungen getan hatte, daß es bei wichtigen Fragen, die im Studentenparlament behandelt wurden, bestimmte Positionen eingenommen hatte. $\ll^{5}$

An der Universität Mainz wurde 1972 noch ein weiterer Fall eines vom VS angeworbenen Abiturienten bekannt: ebenfalls bundeswehrunwillig, ebenfalls durch den VS vom Wehrdienst zurückgestellt, mit dem Auftrag, der SpartakusGruppe Mainz beizutreten und gegen monatliches Honorar von 250 DM einem Verfassungsschutzmann wöchentlich über die Treffpunkte, Namen der Mitglieder und deren Aktivitäten zu berichten. ${ }^{5}$ Was den VS alles interessiert, wissen wir detaillierter von jenem Kieler Studenten Detlef Gritzka, der 1967 vom VS unter dem Decknamen "Grillparzer « in den SDS entsandt wurde, seine Mission jedoch von Anfang an mit dem SDS abstimmte, so daß die Methoden im Detail studiert werden konnten. Gritzka hatte nach folgenden Gliederungspunkten zu berichten:

I. Ort und Zeit der Veranstaltung, Anzahl der Teilnehmer

2. Namen anwesender Personen

3. Bericht über das Referat

4. Verlauf der Diskussion

5. Namen der Beteiligten, die sich bei der Diskussion besonders hervorgetan haben

6. Allgemeiner persönlicher Eindruck ${ }^{6}$

Worauf es vor allem ankam, waren die Namen der engagierten Kommilitonen. Zu Gritzkas Aufgaben gehörte es u. a., Teilnehmer einer Vietnam-Demonstration anhand von Bildern zu identifizieren. Die auf diese Weise zustande kommenden "Erkenntnisse « landen danı in ausführlichen Dossiers, die sowohl von den AfV als auch von der Politischen Polizei geführt werden.

3 Westdeutscher Rundfunk, III. Programm, Kritisches Tagebuch v. 10. 2. 1972, so zitiert in der Dokumentation des Asta der Universität Mainz: Spitzel im Betrieb und an der Universität, Mainz 1972, S. 4 .

- Damm, a. a. O., S. 26; vgl. dazu auch das Tonband-Protokoll eines Spitzel-Anwerbungsgesprächs in : Der Spiegel $21 /$ I $_{973}$, S. $86 \mathrm{f}$. 
Die Einschleusung von Spitzeln in linke Studentenorganisationen ist für die AfV der verschiedensten Bundesländer seit Beginn der Hochschulrevolte gängige Praxis, wobei entweder die Befreiung vom Wehrdienst u. ä. als Lockmittel oder alle möglichen Erpressungsmanöver als Druckmittel verwandt werden. Angesichts der zahlreichen mir bekannt gewordenen Fälle - allein in der Broschüre »So arbeitet der Verfassungsschutz « habe ich über ein Dutzend ausführlich dokumentiert - kann wohl angenommen werden, daß alle linken Hochschulgruppen an allen Hochschulen mindestens von den AfV ständig observiert werden. Gesonderte Erkenntnis-Interessen der Politischen Polizei, des BND, MAD und CIA mögen nach Bedarf hinzukommen. Daß sich diese Überwachung nicht nur auf Studentengruppen, sondern auch auf Professoren, die allgemeinen Studentenausschüsse etc. bezieht, von denen der bayerische Kultusminister Maier äußerte, daß sie "nach Angaben des Verfassungsschutzes« größtenteils »linksradikal « seien und deshalb zerschlagen werden müßten ${ }^{7}$, ist offensichtlich.

Auch außerhalb der Universitäten sind eine Fülle von Fällen bekannt, in denen progressive Personen oder Organisationen von den Staatsschützern überwacht wurden und in denen versucht worden ist, Spitzel einzuschleusen. Das trifft zunächst alle kommunistischen und sozialistischen Gruppierungen, ebenso aber viele Gruppen, die in Einzelfragen der Politik oppositionelle Positionen bezogen, wie etwa die Kampagne für Demokratie und Abrüstung, der Arbeitskreis gegen die Notstandsgesetze, die Internationale der Kriegsdienstgegner, die Hilfsaktion Vietnam, die Gewerkschaften u.v.a.m. Daß nach Ehmke die SPD, nach "Spiegel «- und "Stern «-Veröffentlichungen auch die FDP überwacht wurde, rundet das Bild ab. ${ }^{.}$In diesem Zusammenhang ist auch die Aussage des 20-jährigen Uwe Holtmann vor einem parlamentarischen Untersuchungsausschuß des Kieler Landtags interessant, der zufolge er vom VS angeworben werden sollte, um seinen Religionslehrer und ein SPD-Mitglied zu bespitzeln. ${ }^{\theta}$

Nach einem Bericht im "Stern «10 ruhen neben den ca. 1000 Körperschaftsakten (Unterlagen über Organisationen und Institutionen) pro Großstadt allein bei der Politischen Polizei Bonns 5000, Münchens 10.000 und Berlins über 20.000 Einzeldossiers, in die alles gerät, was über die jeweilige Person oder Organisation von Interesse ist: „Zeugenaussagen, Beobachtungsergebnisse،, Photos, Fingerabdrücke und - so ein 14. K(ommisariat)-Mann - jedermann zugängliche Informationen` wie Zeitungsausschnitte, Flugblätter, Redetexte, kurz alles, was ‘zum Persönlichkeitsbild beiträgt«. Meldungen über Ermittlungsverfahren in Staatsschutz-Delikten müssen die örtlichen Polizeibehörden an eine Zentralkartei des Bundeskriminalamtes und an den VS weiterleiten." Welche Möglichkeiten dadurch im Zeitalter der Computer und zentralen Datenbanken entstehen, ist evident. Als Belastungsmaterial geht alles in die Dossiers ein, was auf progressives Engagement schließen läßt - das geht bis zur Unterschrift unter den Protestaufruf gegen eine NPD-Veranstaltung. ${ }^{10}$

Um die Dossiers mit "Erkenntnissen « zu füllen, wurden die Bezieherkarteien linker Zeitschriften vorübergehend beschlagnahmt ${ }^{11}$, bei der Durchsuchung der psychotherapeutischen Beratungsstelle in Heidelberg "vertrauliche Akten über

\footnotetext{
7 Betrifft: erziehung $3 / 1973$, S. 75 .

Stern I $3 / 4973$, S. 74 .

9 FR 16. 5. 1972.

10 Stern $13 / 1973$, S. 74

11 Vgl. z. B. FR v. 21. 2. 1969, in diesem Fall die des Informationsdienstes "Neue. Kommentare"; vgl. weiter die Wagenbach-Dokumentation über Aktionen der Staatsgewalt gegen die Arbeit des Wagenbach-Verlages, Berlin o. J.
} 
Patienten sowie eine Liste mit Adressen von Patienten mitgenommen « ${ }^{12}$, Demonstranten fotografiert ${ }^{13}$, in der Nähe von Kundgebungsplätzen sogar gewerkschaftlicher Veranstaltungen Autokennzeichen notiert, beim Prozeß gegen Mitglieder der Baader-Meinhof-Gruppe die Ausweise der Zuhörer beim Einlaß in das Gerichtsgebäude fotokopiert ${ }^{14}$, und sogar die Karteien von Krankenkassen-, Unfallversicherungen und Rentenversicherungsanstalten im großen Stile konsultiert. Im Zusammenhang mit der Steiner-Affäre kam weiterhin "ans Licht, daß manchmal unterbezahlte hauptamtliche Parteimitarbeiter zur Aufbesserung ihrer Bezüge als ,Gelegenheitsagenten ‘ auf regionaler Ebene an den Verfassungsschutz vermittelt werden. «15

Wieviele dieser "Erkenntnisse « überhaupt etwas mit der Realität zu tun haben, wieviele allein der Phantasie der Spitzel entspringen, die für ihr Geld Informationen bringen oder der Verfassungsschützer, die ihre Existenzberechtigung nachweisen müssen, kann niemand kontrollieren, da die Dossiers jeder Nachprüfung entzogen sind, was nicht bedeutet, daß sie nicht verwendet werden. So erklärte der Oberpolizeidirektor Hähring von der bayerischen Grenzpolizei, die einem an der Universität Frankfurt eingeschriebenen arabischen Studenten die Einreise in die BRD verwehrte, daß über diesen "Erkenntnisse "vorlägen: "Die Erkenntnisse der VS-Behörden sind zumeist nicht gerichtsverwendbar. Das sollte jedoch nicht bedeuten, daß die Polizei als allgemeine Sicherheitsbehörde auf ihrem ureigensten Gebiet der Prävation nicht auch solche Erkenntnisse verwerten darf. Das Bewußtsein dieser Lage sollte ferner auch für die mögliche Auslegung von Rechtsvorschriften durch Gerichte Beachtung finden. «16 Das bedeutet nichts anderes, als daß die VS-Erkenntnisse zwar nicht für ein Gerichtsverfahren ausreichen, daß sich die Polizei jedoch berechtigt fühlt, auf Grund dieser »nicht verwendbaren « Hinweise die Einreise in die BRD zu verweigern. Diese Logik wurde in einem anderen Fall sogar vom Bundesgerichtshof bestätigt, der einem durch nachweislich falsche Denunziationen des bayerischen LfV geschäftich geschädigten Kaufmann erklärte, daß die AfV auch Nachrichten weitergeben dürfen, die auf ihre Richtigkeit nicht überprüft sind. ${ }^{17}$

Was die Sorgfalt der VS-Recherchen angeht, so liefert der Fall des o.g. arabischen Studenten ein gutes Beispiel. Hähring: »Allein die Lücke zwischen dem Abschluß seines Mathematik-Studiums im Sommer 1963 und dem Beginn seines Volkswirtschafts-Studiums, das er nach Auskunf des Polizeipräsidiums Frankfurt/Main nunmehr im dritten Semester betreibt, macht deutlich, daß er die Zeit wohl nicht an westeuropäischen Maßstäben mißt... T'atsache ist dagegen «, ermittelte die Frankfurter Rundschau, »daß Nizar Abdallah in diesem Zeitraum sein Volkswirtschaft-Studium mit Erfolg abgeschlossen hatte, in seine Heimat zurückgekehrt war, um dort zu arbeiten und dann zurückkam, um in der BRD seinen Doktor zu machen ... Die weiteren Behauptungen der Grenzpolizei, daß nämlich der Doktorand 1968 in Erlangen an einer Tagung der Generalunion Palästinensischer Studenten teilgenommen habe, Vorstandsmitglied der Vereinigung Palästinensischer Studenten gewesen sei, und in dem Ermittlungsverfahren

12 FR 13.7. 1973; ausführliche Darstellung und Nachweise zu den folgenden Praktiken ohne Quellenangabe finden sich in Damm, a.a. O., S. 41-46.

13 Mit welchen eigentümlichen Begründungen Juristen solche VS-Praktiken zu rechtfertigen vermögen, glossiert Ulrich Stascheit in der *Juristenzeitung « s/6/1970, S. I 94.

14 FR I2. 5. 1973.

15 FR 4. 9. 1973.

I6 FR I 3. I. I 973 .

17 Aktenzeichen III ZR 72/65, FR II. 4. 1968. 
gegen El Frangi »als Angehöriger der Al Fatah« erwähnt wurde - von wem, das wird nicht gesagt - werden von Lehrer Horst (einem Freund Nizars - Anm. d. Verf.) in den Bereich der Fabel verwiesen: "Nizar Abdallah war als Syrier nie Mitglied einer palästinensischen Organisation. «18

In den Begründungen für die Massenausweisungen von Arabern im Gefolge der Hysterie nach den Vorfällen bei der Olympiade 1972 tauchte immer wieder die Formel »nach vorliegenden Erkenntnissen «, "nach den vorliegenden Informationen ", "nach den getroffenen Feststellungen « u.ä. auf. ${ }^{19}$ Nach den vorangegangenen Erörterungen wird die Problematik solcher Maßnahmen deutlich.

Es gibt eine lange Tradition solcher Staatsschutzaktionen, die für die Betroffenen oft im Gefängnis, mit der Ausweisung, dem Verlust des Arbeitsplatzes oder der Wohnung u. a. Repressionen endeten. Bereits 1954 wies der Vorsitzende des Bundestags-Ausschusses zum Schutz der Verfassung, Dr. Menzel, auf eine „Fülle» solchen Materials hin. ${ }^{20}$ In einer ganzen Reihe von bekannt gewordenen Fällen erschienen Verfassungschützer beim Arbeitgeber, Vermieter oder Kollegen eines politisch Engagierten, um ihn durch auffällige Erkundigungen, Hausdurchsuchungen oder Vorladungen zur Polizei in Schwierigkeiten zu bringen und einzuschüchtern. Auffällig ist, daß die staatlichen Repressionen sich vorwiegend gegen die politische Linke richten. Das ist nicht nur gegenwärtig beim Berufsverbot so, sondern zeigt sich auch in allen früheren Untersuchungen zur Staatsschutzproblematik in der BRD. Man wäre vielleicht geneigt, diese Tatsache dem Zufall zuzuschreiben, der gerade diese VS-Pannen bekannt werden ließ, korrespondierten sie nicht auffällig mit dem Verhältnis, in dem staatsanwaltliche politische Ermittlungsverfahren gegen links bzw. rechts eingeleitet werden. Für das Jahr 1963 recherchierte Lutz Lehmann, daß nur gegen 177 Rechtsradikale, aber gegen I 0.045 politische Linke ermittelt wurde. Dieses Verhältnis bei der Verfolgung der politischen Linken bzw. Rechten durch die bundesrepublikanischen Staatsschutzinstitutionen ist natürlich nicht zu allen Zeiten gleichbleibend eines von 100:1,7 gegen die politische Linke. Nach allen mir vorliegenden Untersuchungen ist jedoch durch alle Phasen der bundesrepublikanischen Entwicklung die sich in diesem Verhältnis darstellende Tendenz gleichbleibend, daß als "Staatsfeind Nummer I « die politische Linke verfolgt wird.

So war von einem Berufsverbot auch keine Rede, als die NPD in alle Landtage einzog, sondern erst als einige progressive Personen in der Schule u. ä. Institutionen die allenthalben abgegebenen Demokratie-Proklamationen ernst zu nehmen begannen; - wobei selbst nach CDU-Maßstäben »weitaus mehr Rechts- als Linksextremisten im öffentlichen Dienst (beispielsweise) von Rheinland-Pfalz beschäftigt « sind.21

So wird die oben skizzierte Tradition der Unterdrückung der politischen Linken in der BRD durch die gegenwärtige Handhabung des Berufsverbots bestätigt. Einer Dokumentation der demokratischen Aktion sind $4 \mathrm{I}$ Fälle von »Intoleranz gegenüber progressiven Lehrkräften « zu entnehmen, während in den angeführten Bundesländern 3 I prominente Rechtsradikale unangefochten im Staatsdienst lehren.

Repressive Maßnahmen wurden demgemäß gegen demokratisch engagierte SPDund GEW-Mitglieder ebenso ergriffen wie gegen DKP-Mitglieder. Z. B. wurde

18 FR I 3. r. 1973.

$10 \mathrm{FR}$ 28.9. $1972,30.9 .1972$

20 Damm, a. a. O., S. I6-I 8 .

21 FR 29. 9. 1973 
Dietrich Lemke, Sprecher am Studienseminar Hamburg, wie die FR schreibt, "wegen seines Verhaltens am Studienseminar nicht als Beamter, sondern als angestellter Lehrer in den Schuldienst übernommen. Das Juso-Mitglied Lemke hatte sich am Studienseminar für Mitbestimmung eingesetzt. «22 "Siegfried Richter, Lehrer, wurde aus dem Schuldienst entlassen, weil eine Meldung des VS vorlag, der zufolge er Mitglied des Flensburger Sozialistischen Zentrums sein soll ..., Jörg Haslböck, Sozialpädagoge, wurde die Einstellung verweigtert, weil er - wie er selbst bestreitet - Mitglied der DKP sein soll ..., Marion Nürnberg, Junglehrerin, wurde nicht als Beamtin eingestellt, weil sie bei der Wahl zum Studentenparlament für den Spartakus kandidierte und dadurch ihr politisches Engagement unter Beweis gestellt habe ..., Henning Sell, Lehrer, wurde auf Wunsch des VS gekündigt, weil er auf einer NPD-Versammlung mit Mehltüten geworfen habe. $\ll^{23}$

Zum letzten Fall schreibt die FR: "Sell, der in Flensburg Pädagogik studiert hat, wurde von der Hamburger Justizbehörde zunächst als Lehrer für das Jugendgefängnis Vierlande eingestellt. Nach vier Tagen wurde er schon wieder entlassen. Grund: Sell hatte in Flensburg an Demonstrationen teilgenommen. Obwohl ein von der Staatsanwaltschaft gegen ihn eingeleitetes Strafverfahren eingestellt wurde, ist er nach Ansicht der Behörden als Lehter nicht mehr tragbar, weil der VS über den jungen Pädagogen, der sich seit I 969 nicht mehr politisch betätigt, ein Geheindossier angelegt hat. Sell arbeitet heute als Klempner. «24 Ahnlich wie Sell erging es dem 27jährigen Volksschullehrer Reinhard Kordatzki, der sich in Niederbayern vor allem dadurch verdächtig gemacht hatte, daß I. vor seinem Haus eine rote Fahne hing, 2. in seiner Klasse kein Schulgebet gesprochen wurde: "Der Verfassungsschutz übernahm und eruierte prompt: 3 . Kordatzki habe vor Jahren während seines Studiums in München bei den »Roten Zellen « mitgearbeitet. Für Niederbayerns Regierung schloß sich damit die 'Beweis«kette. Daß SPD-Mitglied Kordatzki sentgegen seiner Behauptung، (!) seine damalige Einstellung nicht geändert habe, werde dadurch deutlich, daß er ,freiwillig in einem Haus mit roter Fahne wohne - und daß er in der Schule nicht mehr bete. Die ,Ehrfurcht vor Gott aber, so interpretierte die Regierung auf ihre ganz spezielle Art der Verfassung, gehöre zu den sobersten Bildungsund Erziehungszielen`. Wer dies nicht anerkenne, sei ein Verfassungsfeind. Und Reinhard Kordatzki wurde entlassen. «25

Dabei versuchen die Schulbehörden ähnlich wie die AfV alles, um die Überprüfung und Anfechtung ihrer Maßnahmen zu verunmöglichen - Schulsenator Peter Apel: "Gründe, die uns veranlassen, einen Bewerber zurückzuweisen, werden grundsätzlich nicht angegeben. «20

Im Februar und März 1973 warteten 25 Junglehrer vergeblich auf ihre Einstellung in den Bremer Schuldienst, weil sich die Personenüberprüfung durch den VS, die überall im Öffentlichen Dienst üblich ist, von der nach Aussage von Bürgermeister Koschnik jedoch Lehrer in der Ausbildung angeblich gar nicht betroffen sind, über Monate hinzog. ${ }^{27}$ In Hamburg mußte der Senat eine Entscheidung gegen ein ehemaliges Mitglied des Studentenbundes Spartakus und für einen rechtsradikalen Aktivisten aufheben und genau entgegengesetzt entschei-

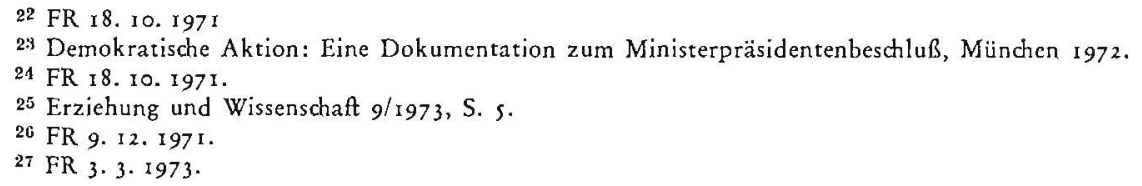


den, weil der VS »in beiden Fällen ... für die Einstellung oder Nichteinstellung wichtige Tatsachen dem Senat nicht unterbreitet hatte. ${ }^{28}$

Wenn angesichts dieser Sachlage die Bundesregierung »im Interesse der Glaubwürdigkeit der freiheitlich-demokratischen Grundordnung " nunmehr durch eine Novellierung des Beamtengesetzes mehr Rechtssicherheit bei der Handhabung des Berufsverbots verspricht ${ }^{29}$, so ist die vorwiegend propagandistische Richtung dieser Proklamation durchsichtig. Denn zum einen werden die tatsächlichen Gründe für die Ablehnung eines Bewerbers im öffentlichen Dienst meist gar nicht angegeben und zum anderen sind die Angaben, die der VS in ein Gerichtsverfahren einbringt, auch vom Gericht nicht nachprüfbar; zumal es in der bundesdeutschen Gerichtsbarkeit die merkwürdige Einrichtung der Zeugen vom Hörensagen gibt, die nicht nachprüfbares VS-Material als Zeugen der Anklage im Prozeß vorbringen, sich jedoch jedem Kreuzverhör der Verteidigung durch Rekurs auf das Staatsgeheimnis oder nicht erteilte Aussagegenehmigung entziehen können.

Im Zusammenhang mit den Praktiken der AfV muß weiter kurz hingewiesen werden auf die Praxis der Telefon- und Postüberwachung und auf den Einsatz von Provokateuren durch die AfV. Nachdem mehr als is Jahre lang AfV, BND und MAD Post und Telefon überwacht hatten, obwohl Art. 1o Grundgesetz zweifelsfrei jeden Eingriff in das Grundrecht des Brief- Post- und Fernmeldegeheimnisses verbot, was 1963 die Telefonabhöraffäre auslöste, wurde denselben Geheimdiensten, die jahrelang das Grundgesetz brachen, durch das Abhörgesetz vom r. I r. I 968 die Möglichkeit gegeben, ihr bisher illegales Treiben nun legal abzuwickeln. Damit wird sehr drastisch eine Tendenz des Parlaments deutlich, Kompetenzüberschreitungen der $\mathrm{AfV}$ nicht durch eine wirksamere Kontrolle, sondern durch Legalisierung des bisher Illegalen zu beantworten. Das gleiche Verfahren ist bei der Ausländer-Überwachung durch die AfV zu beobachten. Während bereits der Bericht des BfV $1969 / 70$ eine I 9 seitige Ausführung über »sicherheitsgefährdende Bestrebungen von Ausländern $1969 / 70$ « enthält, ${ }^{30}$ erhielten die AfV erst 1972 die gesetzlichen Kompetenzen für die AusländerOberwachung.

Bei den von den AfV und der Politischen Polizei eingesetzten »agents provocateurs« handelt es sich um Verfassungsschützer oder vom VS angeworbene Spitzel, die z. B. Demonstranten oder politische Gruppen zuerst zu Straftaten animieren, um sie dann verfolgen zu können, oder die Straftaten begehen, die dann mit großem Propaganda-Aufwand den Linken zugeschoben werden. Ein Beispiel mag dafür der im Baader-Meinhof-Prozeß vom Berliner Senat präsentierte VS-Spitzel Peter Urbach sein, von dem mehrere Zeugen aussagten, daß er Kreisen der Linken Waffen angeboten sowie den Vorschlag gemacht habe, die Freiheitsglocke zu sprengen und daß er Bomben im jüdischen Gemeindehaus und in Wohnungen von Apo-Mitgliedern deponierte, die anschließend zum Teil bei spektakulären Hausdurchsuchungen "gefunden « wurden. ${ }^{31}$ Dabei muß bei einem VS-Spitzel ein Auftrag, zumindest aber eine Absprache mit der Politischen Polizei oder dem Berliner AfV zumindest vermutet werden - will man nicht was nahe liegt, die Art und Weise, wie der Berliner Innensenator Neubauer jede Aufklärung des Gerichts über die "zwielichtige Rolle des V-Mannes « ${ }^{32}$ durch das

28 FR 2. 3. 1973.

20 FR 22. 9. 1973.

30 Bundesinnenministerium: Betr.: Verfassungsschurz 1969/70, S. 53-72.

31 FR 4., II., 27., 28. Mai I 97 I - vgl. auch Dokumentation des Asta Mainz, S. Io.

32 FR II, 5. 1971. 
Entziehen der Aussagegenehmigung etc. verhinderte, als Beweis für die Provokateurrolle Urbachs ansehen. Weitere Fälle ähnlicher Natur habe ich an anderer Stelle dokumentiert. ${ }^{33}$

Die Funktion des VS und der Politischen Polizei könnte man so bestimmen, daß sie zunächst die jeweilige Regierung gegen unliebsame Opponenten, prinzipieller jedoch das kapitalistische Herrschaftssystem gegen fundamentale Demokratisierungsbestrebungen zu schützen haben. Dabei bedienen sich diese Staatsschutzinstitutionen zum einen des Mittels der Uberwachung aller oppositioneller Bestrebungen, deren Ergebnisse sowohl geprüft als auch ungeprüft in hunderttausend von Dossiers aufbewahrt und nach Bedarf aufgefordert oder unaufgefordert weitergegeben werden. Zum anderen ergreifen oder veranlassen sie im großen Umfang direkte Repressionen, die gegenwärtig in der Mehrzahl der Einschüchterung, in nicht unerheblichem Ausmaß jedoch auch der Existenzgefährdung oppositioneller Personen oder Organisationen dienen.

Die BRD ist insofern demokratisch, als die Herrschaft der herrschenden Klasse zumindest der Legitimation bedarf. Dazu gibt es ein engmaschiges System materieller Abhängigkeiten und Bedingungen, die die Entschleierung und Veränderung der herrschenden Verhältnisse erschweren wie etwa die Bedingung, seine Arbeitskraft verkaufen, nach fremdbestimmten Prinzipien arbeiten oder unter den Bedingungen eines kapitalistischen Wohnungsmarktes wohnen zu müssen. Dazu gibt es weiter eine Unmenge von Institutionen, die die Rechtfertigung der bestehenden Zustände leisten: Bewußtseinsindustrie, Kirchen, Wissenschaft, Parlament u. v. a. m. Dort, wo sowohl der materielle wie der ideologische Druck zur Disziplinierung nicht mehr ausreicht, beginnt die Ebene, auf der die Staatsschutzorgane agieren. Der Schwerpunkt ihrer Aktivitäten bezieht sich in Zeiten einer relativ schwachen demokratischen und sozialistischen Bewegung auf deren Oberwachung und Einschüchterung, zu der auch ein gewisses Ausmaß direkter politischer Repression gehört, mit deren Verstärkung zu rechnen ist je brüchiger die o.g. Legitimationen werden, das heißt, je stärker die demokratische Bewegung wird. Für die Strategie der Linken ist an diesem Sachverhalt interessant, daß die Legitimationen objektiv brüchig sind und daß eine Aufklärung über die o.g. Repressionen den Prozeß der Aufklärung über die Natur der bundesrepublikanischen Gesellschaft vorantreiben kann. Das reicht natürlich nicht aus, kann aber ein Element im Kampf um die Demokratisierung dieser Gesellschaft sein.

Dietbelm Damm

${ }^{33}$ Vgl. Damm, a. a. O., S. 21 f. 